\title{
Model for Calculating the Thermophysical Characteristics of Materials According to Multispectral Multi-Temporal Photographic Survey of the Earth's Surface
}

\author{
Igor N. Ischuk* ${ }^{a *}$ Alexey A. Dolgov ${ }^{b}$, \\ Maxim A. Likhachev ${ }^{b}$ and Bogdan K. Telnykh ${ }^{b}$ \\ ${ }^{a}$ Siberian Federal University \\ Krasnoyarsk, Russian Federation \\ ${ }^{b}$ Military Educational and Scientific Center of Air Force \\ "Air Force Academy named after Professor N.E. Zhukovsky \\ and Yu.A. Gagarin» \\ Voronezh, Russian Federation
}

Received 15.09.2020, received in revised form 25.09.2020, accepted 01.10.2020

\begin{abstract}
The article presents a model for calculating the thermophysical parameters of materials according to multispectral multi-temporal photographic survey of the Earth's surface. The analysis of using data on the thermophysical parameters of materials for the identification of monitoring objects is carried out. The numerical estimates of the errors in determining the thermophysical parameters of the tested materials and distribution histograms of the thermophysical parameters of materials according to the field experimental data are also obtained.
\end{abstract}

Keywords: thermophysical parameters, optoelectronic systems, technology of thermal tomography, information characteristic.

Citation: Ischuk I.N., Dolgov A.A., Likhachev M.A., Telnykh B.K. Model for calculating the thermophysical characteristics of materials according to multispectral multi-temporal photographic survey of the earth's surface, J. Sib. Fed. Univ. Eng. \& Technol., 2020, 13(7), 894-906. DOI: 10.17516/1999-494X-0278

(c) Siberian Federal University. All rights reserved

This work is licensed under a Creative Commons Attribution-Non Commercial 4.0 International License (CC BY-NC 4.0).

* Corresponding author E-mail address: boerby76@mail.ru 


\title{
Модель расчета теплофизических параметров материалов по данным многоспектральной разновременной фотографической съемки земной поверхности
}

\author{
И.Н. Ищук ${ }^{\mathrm{a}}$, А.А. Долгов ${ }^{0}$, \\ М.А. Лихачев ${ }^{\tilde{0}}$, Б.К. Тельных ${ }^{\sigma}$ \\ ${ }^{a}$ Сибирский федеральныий университет \\ Российская Федераџия, Красноярск \\ ${ }^{6}$ Военный учебно-научный иентр Военно-воздушных сил \\ «Военно-воздушная академия \\ имени профессора Н.Е. Жуковского и Ю.А. Гагарина» \\ Российская Федерация, Воронеж
}

Аннотация. В статье представлена модель расчета теплофизических параметров материалов по данным многоспектральной разновременной фотографической съемки земной поверхности. Проведен анализ использования данных теплофизических параметров материалов для идентификации объектов мониторинга; получены численные оценки погрешности определения теплофизических параметров испытуемых материалов, гистограммы распределения теплофизических параметров материалов по данным натурного эксперимента.

Ключевые слова: теплофизические параметры, оптико-электронные системы, технология тепловой томографии, информационный признак.

Цитирование: Ищук, И.Н. Модель расчета теплофизических параметров материалов по данным многоспектральной разновременной фотографической съемки земной поверхности / И.Н. Ищук, А.А. Долгов, М.А. Лихачев, Б.К. Тельных // Журн. Сиб. федер. ун-та. Техника и технологии, 2020. 13(7). С. 894-906. DOI: 10.17516/1999-494X-0278

\section{Введение}

Развитие оптико-электронных систем (ОЭС) расширило их возможности для применения в различных сферах, в том числе в задачах воздушного мониторинга. Это позволило осуществлять аэрофотосъемку местности и объектов в широком диапазоне длин волн, особенно в инфракрасном (ИК) диапазоне длин волн.

Основная проблема идентификации объектов по аэрофотоснимкам и видеоизображениям в видимом и ИК-диапазоне длин волн заключается в выборе необходимых информационных признаков, которые зачастую не в полной мере раскрывают свойства объекта, что в конечном счете может привести к его неправильному распознаванию.

Известно, что качество аэрофотоснимков в ИК-диапазоне длин волн зависит от целого комплекса условий, одним из которых являются теплофизические параметры объектов воздушного мониторинга. Немаловажную роль играют и метеорологические условия района наблюдения.

Решение задачи определения теплофизических параметров (ТФП) материалов позволит создать новый идентификационный признак, что даст возможность достовернее определять квазистационарные (стационарные), пассивные (активные) объекты, включая скрытые. 


\section{1. Анализ использования данных теплофизических параметров материалов для распознавания типов объектов}

Решение задачи оценки теплофизических параметров материалов по данным разновременных инфракрасных изображений обеспечивает поиск малозаметных объектов и объектов с изменяющимися видовыми признаками $[1,2]$, дальнейшее развитие которых связано с построением нового идентификатора объектов. Рассмотрим идентификатор объектов с учетом их ТФП.

Идентификатор «Фон, объекты района коррекции». Идентификатор предназначен для распознавания типов объектов по материалу на разновременных многоспектральных изображениях по результатам съемки объектов в районе дистанционного мониторинга с применением многоспектральной ОЭС.

Типы объектов могут быть представлены линейными, точечными и площадными объектами, зафиксированными на разновременных фотоснимках в инфракрасном и видимом диапазонах длин волн и представленными на местности стационарными объектами («инженерные коммуникации», «здание» и «сооружение», «теплотрасса» и другими), или квазистационарными объектами, которые в течение наблюдения не изменили своего местоположения, а также имеющими внутренний источник тепла («автомобиль», «самолет», «вертолет», «железнодорожная платформа» и др.).

Параметрами идентификатора по типу объектов являются индивидуальные ТФП этих объектов. В соответствии с этими данными формируются дополнительные параметры объектов (фонов), входящих в идентификатор «Фон, объекты района коррекции».

«Однородная изотропная среда» - категория идентификатора «Фон, объекты района коррекции». Координаты пикселей изображения объекта соответствуют фону модели пространственного распределения ТФП объектов.

В качестве параметра, характеризующего его ТФП, может быть использована тепловая активность материала (тепловая инерция) $I$, определяемая в соответствии с выражением [3]:

$$
I=\sqrt{\lambda C \rho},
$$

где $\lambda$ - теплопроводность [Вт $\left.\cdot \mathrm{M}^{-1} \mathrm{~K}^{-1}\right]$; $\mathrm{C}-$ удельная теплоемкость [Дж $\left.\cdot \mathrm{\kappa}^{-1} \mathrm{~K}^{-1}\right] ; \rho-$ плотность $\left[\kappa^{-3}\right]$ материалов и сред.

Объектам (фонам) присваивают оценку значения ТФП или коэффициента тепловой активности (тепловой инерции $I$ ), и на основании нормировочной шкалы задают соответствующий вид материала [3, 4]:

«материал сверхмалой теплопроводности»- $(\lambda \leq 0.029) \mathrm{BT}^{\cdot} \mathrm{M}^{-1} \mathrm{~K}^{-1}$;

«теплоизоляционный материал» $-(0.029<\lambda \leq 0.175) \mathrm{BT} \cdot \mathrm{M}^{-1} \mathrm{~K}^{-1}$;

«конструкционно-теплоизоляционный материал» - $(0.175<\lambda \leq 0.7) \mathrm{BT} \cdot \mathrm{M}^{-1} \mathrm{~K}^{-1}$;

«конструкционный материал» - $(0.7<\lambda \leq 1.7) \mathrm{BT}^{\cdot} \mathrm{M}^{-1} \mathrm{~K}^{-1}$;

«материал - природный камень» - $(1.7<\lambda \leq 3.5) \mathrm{BT}^{\cdot} \mathrm{M}^{-1} \mathrm{~K}^{-1}$;

«материал - металлы» - $(\lambda>3.5)$ Вт $\cdot \mathrm{M}^{-1} \mathrm{~K}^{-1}$,

с возможным указанием типа материала (табл. 1, по данным свода правил по проектированию и строительству [4]). Классы «нефть», «снег», «вода», «лед» идентифицируют по данным предварительной классификации изображения в видимом диапазоне длин волн [4]. 
Таблица 1. Типы материалов и их ТФП [4]

Table 1. Materials and their thermophysical characteristics

\begin{tabular}{|c|c|c|c|c|c|c|c|c|c|}
\hline \multirow[t]{2}{*}{ Материал } & \multicolumn{2}{|c|}{$\begin{array}{c}\rho, \\
\kappa \Gamma \cdot \mathrm{M}^{-3}\end{array}$} & \multicolumn{2}{|c|}{$\begin{array}{c}\lambda, \\
\mathrm{BT}^{\prime} \cdot \mathrm{M}^{-1} \mathrm{~K}^{-1}\end{array}$} & \multicolumn{2}{|c|}{$\begin{array}{c}C, \\
\text { Дж } \kappa^{-1} \Gamma^{-1} K^{-1}\end{array}$} & \multicolumn{2}{|c|}{$\begin{array}{c}I, \\
\text { Дж } \cdot \mathrm{M}^{-2} \mathrm{~K}^{-1} \mathrm{c}^{-1 / 2} \\
\end{array}$} & \multirow{2}{*}{$\begin{array}{c}\overline{\mathrm{I}} \\
\text { сред. }\end{array}$} \\
\hline & Min & Max & Min & Max & Min & Max & Min & Max & \\
\hline Пенопласт & 65 & 125 & 0,031 & 0,05 & 1150 & 1260 & 48 & 88 & 68 \\
\hline Минералвата & 125 & 200 & 0,06 & 0,08 & 820 & 840 & 78 & 115 & 96 \\
\hline Щебень & 200 & 600 & 0,064 & 0,11 & 820 & 860 & 102 & 238 & 170 \\
\hline Полистеролбетон & 200 & 600 & 0,065 & 0,145 & 900 & 1060 & 108 & 303 & 205 \\
\hline Керамзит & 800 & 1500 & 0,16 & 0,4 & 750 & 840 & 309 & 709 & 509 \\
\hline Дерево & 600 & 750 & 0,15 & 0,23 & 2600 & 2700 & 483 & 682 & 582 \\
\hline Кирпич & 1000 & 1500 & 0,35 & 0,64 & 800 & 880 & 529 & 919 & 724 \\
\hline Песок & 1400 & 1500 & 0,3 & 1,2 & 700 & 840 & 542 & 1229 & 885 \\
\hline Грунт & 1400 & 1500 & 0,4 & 2,1 & 800 & 860 & 669 & 1645 & 1157 \\
\hline Бетон & 1800 & 2500 & 0,2 & 1,75 & 710 & 880 & 505 & 1962 & 1233 \\
\hline Асфальт & 1100 & 2110 & 0,7 & 1,05 & 1700 & 2100 & 1144 & 2156 & 1650 \\
\hline Базальт & 2600 & 3000 & 3 & 3,5 & 830 & 850 & 2544 & 2987 & 2765 \\
\hline Металл & 2500 & 7900 & 15 & 120 & 120 & 900 & 2121 & 29209 & 15665 \\
\hline Нефть & 730 & 1040 & 0,14 & 0,15 & 2000 & 2100 & 452 & 572 & 512 \\
\hline Снег & 120 & 400 & 0,1 & 0,5 & 2000 & 2100 & 154 & 648 & 401 \\
\hline Вода & 980 & 1000 & 0,6 & 0,7 & 4190 & 4200 & 1569 & 1714 & 1641 \\
\hline Лед & 910 & 920 & 2 & 2,2 & 2100 & 2150 & 1954 & 2086 & 2020 \\
\hline
\end{tabular}

Например: фон «дорога» - «однородная изотропная среда», «конструкционный материал» - «асфальт», оценка $\hat{\lambda} \approx 1,1 \mathrm{Bm} \cdot \mathrm{M}^{-1} \mathrm{~K}^{-1}, \hat{I} \approx 1200$ Дж$\cdot \mathrm{M}^{-2} \mathrm{~K}^{-1} \mathrm{c}^{-1 / 2}$.

«Квазистационарный надповерхностный пассивный объект»-категория в идентификатоpe «Фон, объекты района коррекции», область координат которого соответствует объектам, находящимся над поверхностью фона модели пространственного распределения ТФП объектов. Объектам присваивается оценка значения коэффициента тепловой активности, и на основании нормировочной шкалы задается соответствующий вид материалов: «материал сверхмалой теплопроводности», «теплоизоляционный материал», «конструкционно-теплоизоляционный материал», «конструкционный материал», «материал - природный камень», «материал - металлы», с возможным указанием типа материала, например «пенопласт», «бетон», «асфальт», «вода», «нефть», «металл» и иные материалы.

Например: «Квазистационарный надповерхностный пассивный объект» - «автомобиль» - «материал - металлы» - типа материала «металл», оценка $\hat{\lambda} \approx 13,1 \mathrm{Bm} \cdot \mathrm{M}^{-1} \mathrm{~K}^{-1}$, $\hat{I} \approx 10200$ Дж $\cdot \mathrm{M}^{-2} \mathrm{~K}^{-1} \mathrm{c}^{-1 / 2}$.

«Квазистационарный надповерхностный активный объект» - категория в идентификаторе «Фон, объекты района коррекции», который соответствует надповерхностным объектам модели пространственного распределения ТФП объектов, но имеющий внутренний источник тепла. Данной категории объектов присваивается оценка значения коэффициента тепловой ак-

$$
-897-
$$


тивности, и на основании нормировочной шкалы с учетом наличия внутреннего источника тепла задается соответствующий вид материалов: «материал сверхмалой теплопроводности», «теплоизоляционный материал», «конструкционно-теплоизоляционный материал», «конструкционный материал», «материал - природный камень», «материал - металлы», с указанием типа материала, например «пенопласт», «песок», «бетон», «асфальт», «металл», а также с уточнением физического состояния объектов мониторинга («автомобиль» - «автомобиль с запущенным ДВС»).

Например: «Квазистационарный надповерхностный активный объект» - «автомобиль с запущенным ДВС» - «материал - металлы» - типа материала «металл», оценка $\hat{\lambda} \approx 50,1 \mathrm{Bm} \cdot \mathrm{M}^{-1} \mathrm{~K}^{-1}, \hat{I} \approx 13200$ Дж$\cdot \mathrm{M}^{-2} \mathrm{~K}^{-1} \mathrm{c}^{-1 / 2}$.

«Скрытый пассивный квазистационарный объект» - категория в идентификаторе «Фон, объекты района коррекции», область координат пикселей которого соответствует объектам под поверхностью фона модели пространственного распределения ТФП объектов. Объектам присваивается оценка значения коэффициента тепловой активности, и на основании нормировочной шкалы задается соответствующий класс материалов: «теплоизоляционный материал», «конструкционный материал», «материал - металлы», с указанием типа материала, например «пенопласт», «бетон», «вода», «металл» и иные.

Например: «Скрытый пассивный квазистационарный объект» - «инженерные коммуникации» - «теплоизоляционный материал» - типа материала «минералвата», оценка $\hat{\lambda} \approx 0,07 \mathrm{Bm} \cdot \mathrm{M}^{-1} \mathrm{~K}^{-1}, \hat{I} \approx 90$ Дж$\cdot \mathrm{m}^{-2} \mathrm{~K}^{-1} \mathrm{c}^{-1 / 2}$.

«Скрытый активный квазистационарный объект»- категория в идентификаторе «Фон, объекты района коррекции», который соответствует объектам под поверхностью фона и имеющим внутренний источник тепла модели пространственного распределения ТФП объектов. Объектам присваивается оценка значения коэффициента тепловой активности, и на основании нормировочной шкалы задается соответствующий класс материалов: «теплоизоляционный материал», «конструкционный материал», «материал - металлы», с возможным указанием типа материала, например «пенопласт», «вода», «нефть», «металл» и иные материалы с уточнением их физического состояния: «инженерные коммуникации с горячим водоснабжением», «горящий торф» и т.д.

Например: «Скрытый активный квазистационарный объект» - «инженерные коммуникации с горячим водоснабжением» - «теплоизоляционный материал» - типа материала «минералвата», оценка $\hat{\lambda} \approx 0,07 \mathrm{Bm} \cdot \mathrm{M}^{-1} \mathrm{~K}^{-1}, \hat{I} \approx 90$ Дж$\cdot \mathrm{M}^{-2} \mathrm{~K}^{-1} \mathrm{c}^{-1 / 2}$.

\section{2. Модель и алгоритм построения пространственного распределения ТФП материалов}

Пространственное распределение температурных полей для твердых изотропных сред может быть описано на основе закона Фурье о пропорциональности вектора плотности теплового потока $\theta$ антиградиенту температуры в соответствии с выражением

$$
\theta=-\lambda \operatorname{grad} T
$$

где $-\lambda$ коэффициент теплопроводности изотропной среды, имеющий постоянное значение в различных направлениях принятой системы координат.

$$
\text { - } 898-
$$


Вектор плотности теплового потока в анизотропных средах характеризуется различными значениями теплопроводности при движении в различных направлениях.

Поэтому уравнение (2) для анизотропной среды можно записать в матричной форме:

$$
-\theta=\left(\begin{array}{l}
-\theta_{x} \\
-\theta_{y} \\
-\theta_{z}
\end{array}\right)=\left(\begin{array}{lll}
\lambda_{x x} & \lambda_{x y} & \lambda_{x z} \\
\lambda_{y x} & \lambda_{y y} & \lambda_{y z} \\
\lambda_{z x} & \lambda_{z y} & \lambda_{z z}
\end{array}\right)\left(\begin{array}{l}
\partial T / \partial x \\
\partial T / \partial y \\
\partial T / \partial z
\end{array}\right)=\Omega \operatorname{grad} T
$$

где $\Omega=\left(\begin{array}{lll}\lambda_{x x} & \lambda_{x y} & \lambda_{x z} \\ \lambda_{y x} & \lambda_{y y} & \lambda_{y z} \\ \lambda_{z x} & \lambda_{z y} & \lambda_{z z}\end{array}\right)$ - тензор теплопроводности анизотропной среды.

Граничные условия теплового баланса на верхней границе среды и сопряжения слоистых сред для случая неидеального теплового контакта между двумя поверхностями (контактные поверхности разделены тонкой прослойкой) будут выглядеть следующим образом [6]:

$$
\begin{gathered}
-\left.C^{*} \rho^{*} \alpha_{K} \frac{\partial T}{\partial z}\right|_{z=0}-\left.L_{\rho} \alpha_{K} \frac{\partial v}{\partial z}\right|_{z=0}-\left.\lambda_{1}(T, q) \frac{\partial T}{\partial z}\right|_{z=0}-B_{0}^{*}=Q-\left.C_{v} \frac{\partial Q_{v} T}{\partial z}\right|_{z=0}-\left.L_{\rho} \frac{\partial Q_{v}}{\partial z}\right|_{z=0} ; \\
\left.\lambda_{j}(T, q) \frac{\partial T}{\partial z}\right|_{z=-\sum_{i=1}^{j} h_{i}-(j-1) \Delta h}=\frac{1}{R_{T}}\left(\left.\frac{\partial T}{\partial z}\right|_{z=-\sum_{i=1}^{j} h_{i}-j \Delta h}-\left.\frac{\partial T}{\partial z}\right|_{z=-\sum_{i=1}^{j} h_{i}-(j-1) \Delta h}\right) ; \\
\left.\lambda_{j}(T, q) \frac{\partial T}{\partial z}\right|_{z=-\sum_{i=1}^{j} h_{i}-(j-1) \Delta h}=\left.\lambda_{j+1}(T, q) \frac{\partial T}{\partial z}\right|_{z=-\sum_{i=1}^{j} h_{i}-j \Delta h} ; \\
\left.T_{0}\right|_{z=0}=\varphi_{0}(x, y) ; \\
\left.T_{H}\right|_{z=-H}=\varphi_{1}(x, y),
\end{gathered}
$$

где $C^{*}$ - удельная изобарная теплоемкость воздуха; $\rho^{*}-$ плотность воздуха; $\alpha_{K}-$ коэффициент турбулентности (коэффициент теплоотдачи); v - массовая доля водяного пара; $L_{\rho}$ - удельная теплота парообразования; $Q$ - плотность теплового потока; $C_{v}$ - удельная теплоемкость водяного пара; $B_{0}^{*}=\varepsilon \sigma\left(T_{0}^{4}-\varepsilon_{\mathrm{a}} T_{\mathrm{a}}^{4}\right)-$ эффективное излучение верхнего слоя поверхности; $\sigma=5,67 \cdot 10^{-8}$ $\left[\mathrm{BT} \cdot \mathrm{M}^{-2} \cdot K^{-4}\right]-$ постоянная Стефана-Больцмана; $R_{T}$ - контактное сопротивление (контактная проводимость), $\left[\mathrm{BT}^{-1} \cdot \mathrm{M} \cdot K\right] ; \varphi_{0}(x, y), \varphi_{1}(x, y)$ - известные функции начального распределения температур; $Q_{v}$ - поток тепла, участвующий в конвективном теплообмене посредством движения воды и водяного пара внутри пористой неоднородной среды; $h_{i}-$ толщина і-го слоя среды; $\Delta h$ - толщина контактного слоя.

Зависимости значений теплопроводности и теплоемкости среды от ее температуры и влажности описывают эмпирическими выражениями $[6,7]$ :

$$
\begin{aligned}
& \lambda(T)=\lambda_{0}\left[1+g_{0}\left(T-T_{0}\right)\right], \\
& \lambda(q)=g_{1}+g_{2} q+g_{3} q^{\frac{1}{2}}, \\
& C(T)=r_{0}+r_{1} T+r_{2} T^{2}+r_{-2} T^{\frac{1}{2}}, \\
& C(q)=C_{0}(1+0,01 q / 1+0,01 q),
\end{aligned}
$$

где $\lambda_{0},\left[\right.$ Вт $\left.\cdot \mathrm{M}^{-1} \cdot K^{-1}\right]$ - значение теплопроводности при температуре $T_{0},[\mathrm{~K}] ; q$ - весовая влажность среды, [\%]; $g_{0},\left[K^{-1}\right] ; g_{1},\left[\mathrm{BT} \cdot \mathrm{M}^{-1} \cdot K^{-1}\right] ; g_{2},\left[\mathrm{BT} \cdot \mathrm{M}^{-1} \cdot K^{-1}\right] ; g_{3},\left[\mathrm{BT} \cdot \mathrm{M}^{-1} \cdot K^{-1}\right] ; r_{0}$, [Дж $\left.\cdot \mathrm{K}^{-1} \cdot K^{-1}\right]$; 
$r_{1},\left[\right.$ [ж $\left.\cdot \kappa \Gamma^{-1} \cdot K^{-2}\right] ; r_{2}$,[Дж $\left.\cdot \kappa \Gamma^{-1} \cdot K^{-3}\right] ; r_{-2}$,[Дж $\left.\cdot \kappa \Gamma^{-1} \cdot K\right]-$ эмпирические коэффициенты, зависящие от состава материала и среды.

Для построения пространственного распределения ТФП материалов предложен алгоритм, отличительной особенностью которого является построение модели распределения ТФП исследуемых объектов, обеспечивающая корректное задание граничных условий сопряжения слоистых сред и позволяющая получить численные оценки ТФП материалов и сред, принадлежащих множеству допустимых значений [8].

Порядок построения пространственного распределения ТФП заключается в выполнении следующих этапов.

Этап 1. Загрузка видимого изображения и разновременных изображений в ИК-диапазоне длин волн для дальнейшей обработки. Съемка изображений объектов района коррекции производится в надир с заданной периодичностью:

в видимом диапазоне длин волн ряд снимков, следующих в хронологическом порядке, произведенных в светлое время суток через одинаковые промежутки времени в течение временного интервала $\Delta t_{\text {вид }}<\tau$, где $\tau-$ время дистанционного мониторинга заданного района исследуемой поверхности;

в ИК-диапазоне длин волн ряд снимков, следующих в хронологическом порядке, произведенных в течение суток через одинаковые промежутки времени (максимальных значений функции источника тепла) [5], через равные промежутки времени в течение временного интервала съемки $\Delta t_{\text {Ик }}<<\tau$.

Этап 2. Расчет поля термодинамических температур согласно выражению

$$
\widetilde{T}=\sqrt[4]{\frac{\widetilde{T}_{r}^{4}-(1-\varepsilon) T_{a}^{4}}{\varepsilon}},
$$

где $\widetilde{T}_{r}$ - значение радиационной температуры исследуемой поверхности; $\varepsilon$ - коэффициент излучательной способности поверхности, рассчитываемый в соответствии с методикой определения коэффициента теплового излучения для спектрального диапазона, в котором работает тепловизионный приемник (ТПВП); $T_{a}$ - значение температуры окружающей среды.

Этап 3. Построение кубоидов изображений видимого и ИК-диапазонов длин волн путем выполнения операций фотограмметрической обработки, заключающихся в преобразовании метрической и спектральной информации снимка в геометрическую и семантическую информацию об объектах фотограмметрической съемки [9].

Этап 4. Построение модели фоно-целевой обстановки района коррекции на основе совместной обработки изображений видимого и ИК-диапазонов длин волн с целью корректного задания граничных условий взаимного сопряжения слоистых сред и расчета пространственного распределения температурных полей района коррекции.

Этап 5. Поиск стохастически регуляризированного по принципу невязки решения коэффициента обратной задачи теплопроводности (КОЗТ) с применением генетического алгоритма оптимизации, обеспечивающее получение численных оценок ТФП материалов и сред поверхности района коррекции, принадлежащих компактному множеству допустимых значений, для всего растра ИК-изображений, и построение ТТ.

$$
-900-
$$


Этап 6. Вывод трехслойной ТТ объектов наблюдения и фона.

\section{3. Методика проведения эксперимента}

Программа натурного эксперимента заключается в получении численных оценок погрешности определения теплофизических параметров испытуемых материалов по данным многоспектральной разновременной съемки, полученных с помощью ОЭС беспилотного летательного аппарата мультироторного типа с использованием специального программного обеспечения (СПО) «HeatTomogramms».

Контрольный эксперимент включает восемь разновременных изображений в ИК и одно изображение в видимом диапазонах длин волн, на которых отражены испытуемые материалы размерами не менее 120х135 пикселей, взаимно привязанных друг к другу (рис. 1).

Эталонные материалы и их теплофизические свойства представлены в табл. 2.

Оценка погрешности определения теплофизических параметров пяти испытуемых материалов заключается в проведении косвенных измерений ТФП 100 раз подряд в заданных координатах с использованием методики работы с СПО «НеatTomogramms». Для оценки систематической составляющей измерений теплопроводности применяют модуль разности математического ожиданий результатов 100 измерений теплопроводности и среднего значения теплопроводности для заданного типа эталонного материала, получаемого табличным способом по данным табл. 2.

Целью эксперимента являлось подтверждение возможности производить идентификацию объектов мониторинга по показателям теплофизических свойств (параметров) материалов по разновременным изображениям ИК-диапазона.

Численные расчеты проводили в программно-ориентированной среде MATLAB R2014a на ЭBM (процессор AMD Opteron (2.5 ГГц), объем оперативной памяти 128 Гб, жесткий диск SSD 1 Тб, видеокарта NVIDIA GeForce GTX 7806 Гб), позволяющей поддерживать до 80 потоков параллельных вычислений СПО «HeatTomogramms».

Для расчета ТФП материалов применяли генетический алгоритм оптимизации с заданным порогом целевой функции, равным 2. Во время работы СПО получены графики измене-

Таблица 2. Теплофизические свойства испытуемых материалов [4]

Table 2. Thermophysical characteristics of tested materials

\begin{tabular}{|c|c|c|c|c|c|c|c|c|c|}
\hline \multirow{3}{*}{ Материал } & \multirow{2}{*}{\multicolumn{2}{|c|}{ Координаты }} & \multicolumn{7}{|c|}{ Теплофизические свойства } \\
\hline & & & \multicolumn{3}{|c|}{$\begin{array}{c}\lambda, \\
\mathrm{BT} \cdot \mathrm{M}^{-1} \cdot K^{-1}\end{array}$} & \multicolumn{2}{|c|}{$\begin{array}{c}\mathrm{C}, \\
\text { Дж } \cdot \mathrm{\kappa}^{-1} \cdot K^{-1}\end{array}$} & \multicolumn{2}{|c|}{$\begin{array}{c}\rho, \\
\text { кГ } \cdot \mathbf{M}^{-3}\end{array}$} \\
\hline & $\mathrm{X}$ & $\mathrm{Y}$ & Min & Max & $\begin{array}{c}\text { среднее } \\
\text { значение }\end{array}$ & Min & Max & Min & $\operatorname{Max}$ \\
\hline Пенопласт & 24 & 43 & 0.01 & 0.04 & 0,025 & 1450 & 1550 & 10 & 50 \\
\hline Металл & 23 & 50 & 30 & 50 & 40 & 450 & 550 & 7000 & 8000 \\
\hline Бетон & 80 & 91 & 0,2 & 1,75 & 0,975 & 710 & 880 & 1800 & 2500 \\
\hline Песок & 71 & 13 & 0,3 & 1,2 & 0,75 & 700 & 840 & 1400 & 1500 \\
\hline Грунт & 30 & 90 & 0,3 & 3,0 & 1,65 & 800 & 900 & 1500 & 1700 \\
\hline
\end{tabular}

$$
-901-
$$




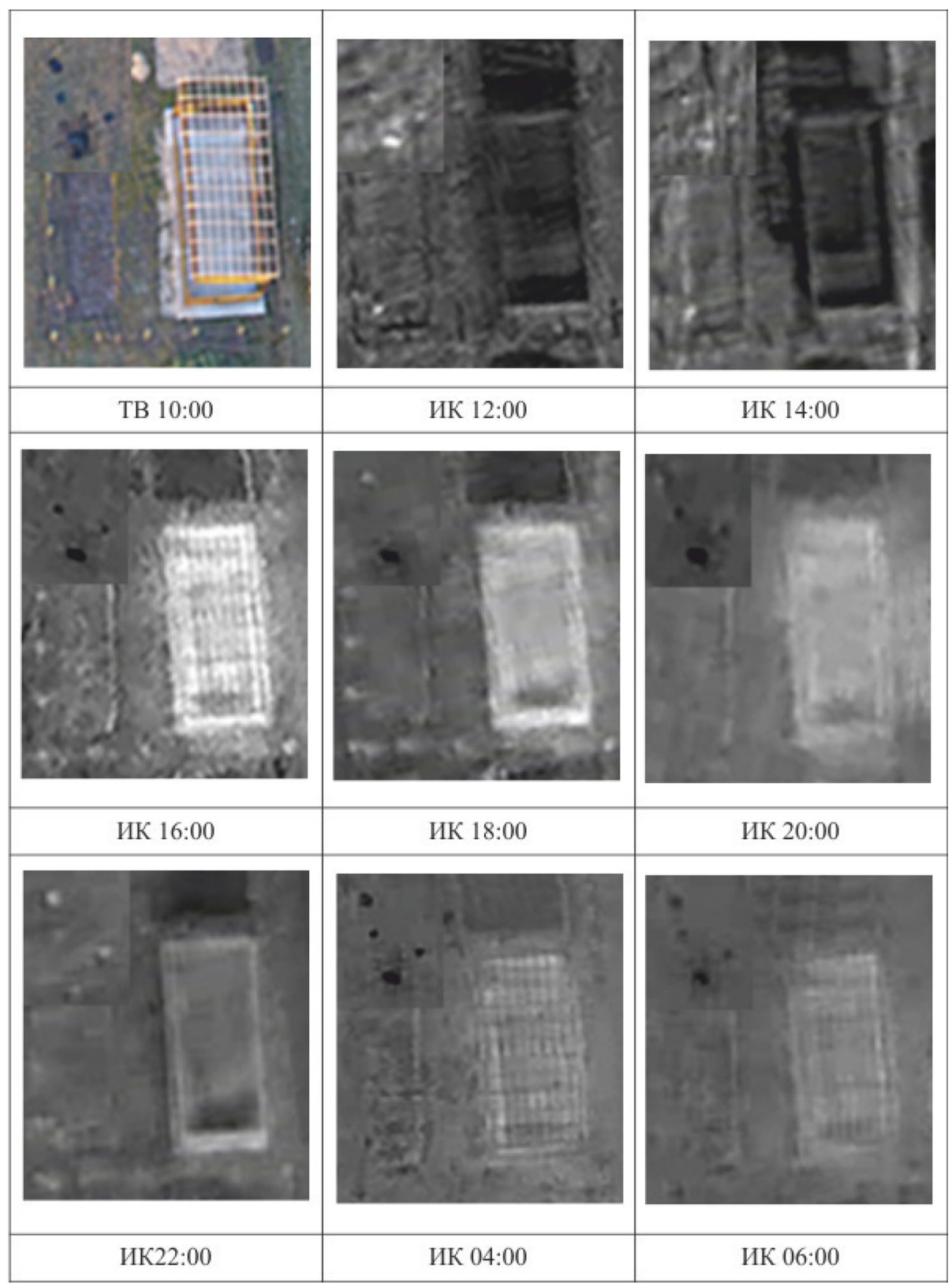

Рис. 1. Разновременные изображения испытуемых материалов в ИК- и видимом диапазонах длин волн Fig. 1. Multi-time TV and IR images of tested materials

ния температур в точках эталонных материалов, построенные по данным съемки с ОЭС БпЛА (красный цвет), и графики температур, построенные в процессе работы генетического алгоритма (ГА) (синий цвет) (рис. 2, 3).

Из данных графиков видно, что на этапе оптимизации значения квадратичного функционала невязки эталонных материалов не превысили порог целевой функции.

По результатам численных расчетов натурного эксперимента построены гистограммы распределения ТФП материалов эталонов (рис. 4).

$$
-902-
$$




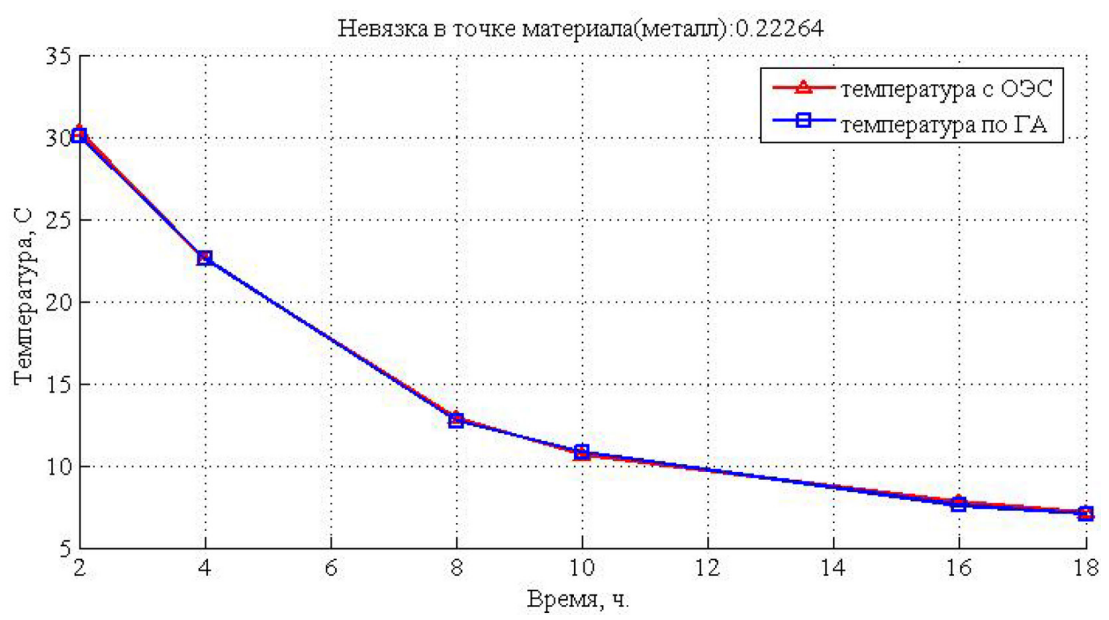

Рис. 2. График изменения температуры в точках испытуемого материала (металл)

Fig. 2. The plot of temperature distribution in points of the tested material (metal)

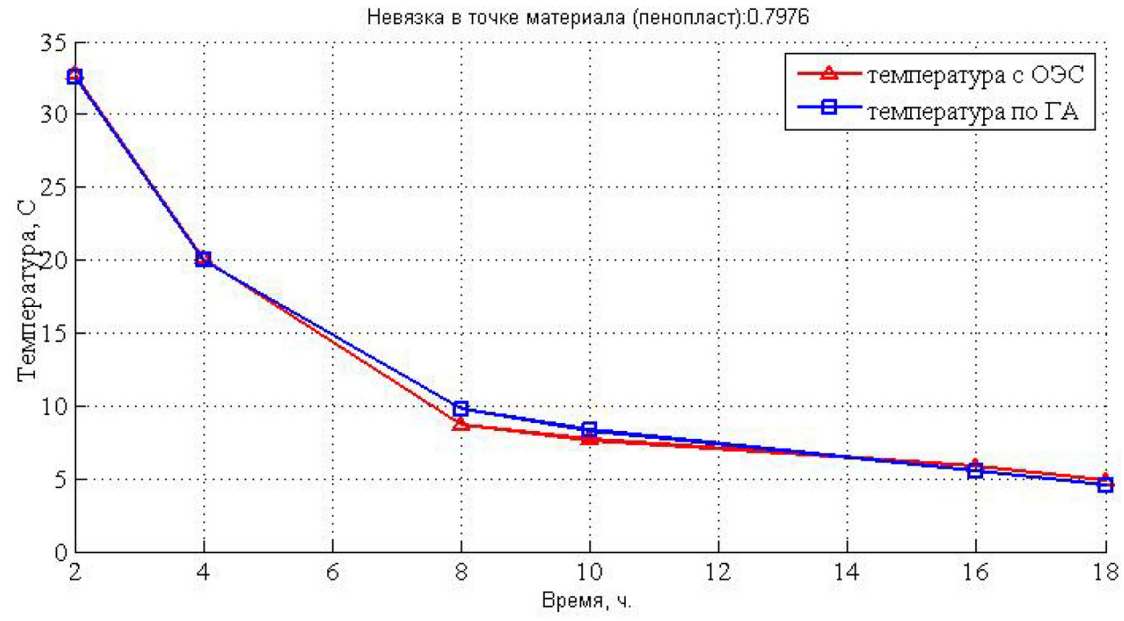

Рис. 3. График изменения температуры в точках испытуемого материала (пенопласт)

Fig. 3. The plot of temperature distribution in points of the tested material (polyfoam)

По гистограммам теплофизических параметров, отражающих $I$ - тепловую активность и a - температуропроводность, прослеживается распределение, близкое к нормальному закону.

Доверительный интервал, характеризующий точность измерения с заданной вероятностью $P=0.90$, для исследуемых материалов согласно нормальному закону распределения для I и $а$ отражен в табл. 3 .

\section{Вывод}

Таким образом, экспериментально подтверждена возможность использования предложенной модели расчета теплофизических параметров материалов по данным многоспектральной разновременной фотографической съемки земной поверхности, которая позволяет распозна- 


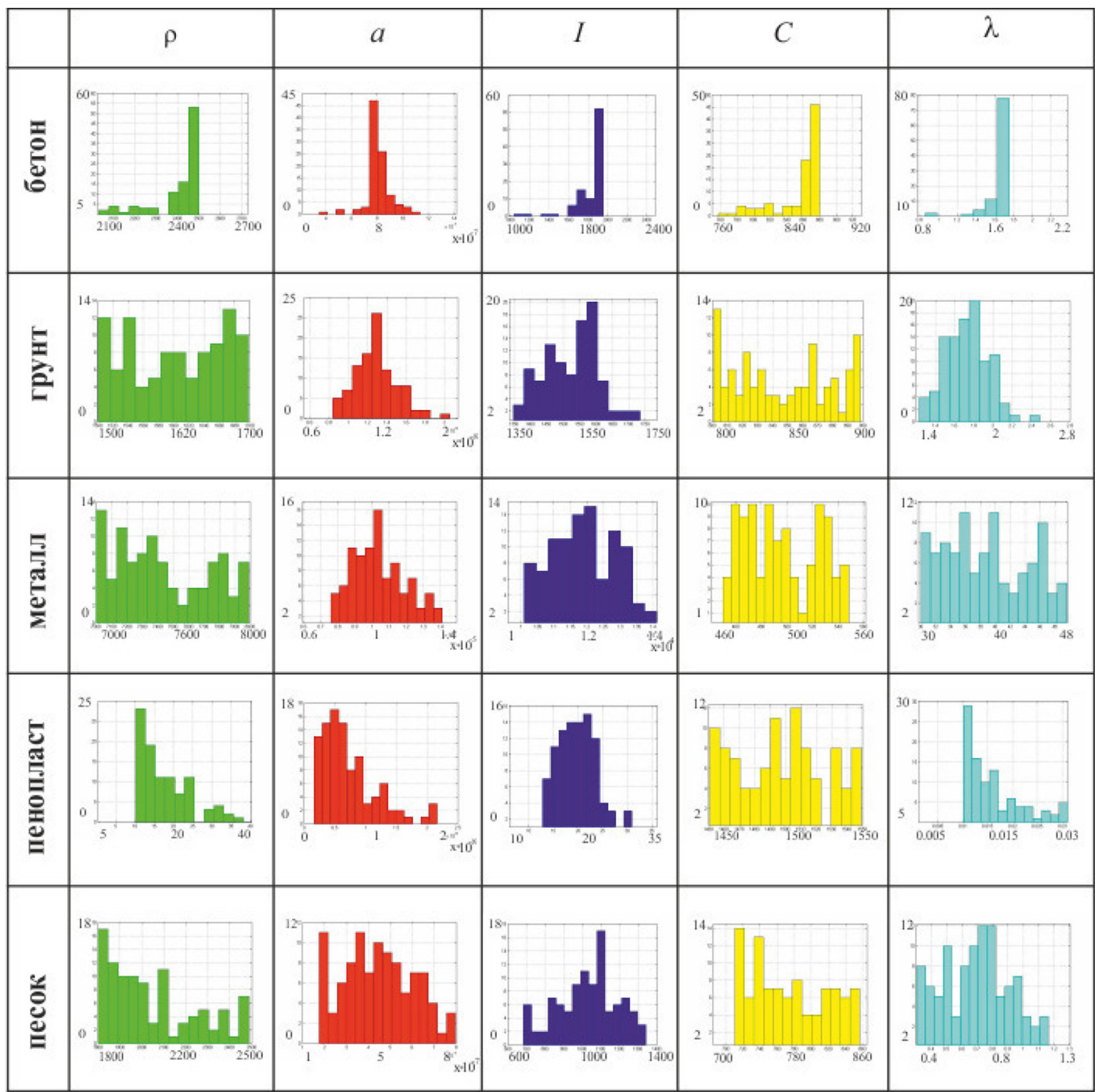

Рис. 4. Гистограммы распределения ТФП испытуемых материалов, полученных по данным натурного эксперимента

Fig. 4. The histogram distribution of thermophysical parameters of tested materials on the field experimental data

Таблица 3. Доверительный интервал распределения I, а испытуемых материалов

Table 3. Confidence interval of distribution I, a tested materials

\begin{tabular}{|c|c|c|}
\hline МАТЕРИАЛ & $\begin{array}{c}\text { Доверительный интервал } \\
a=\bar{a} \pm \sigma_{\xi}\end{array}$ & $\begin{array}{c}\text { Доверительный интервал } \\
I=\bar{I} \pm \sigma_{\xi}\end{array}$ \\
\hline Бетон & $7,91 \cdot 10^{-7} \pm 1,98719 \cdot 10^{-13}$ & $1802,049587 \pm 60,8279$ \\
\hline Грунт & $1,28 \cdot 10^{-6} \pm 2,71477 \cdot 10^{-12}$ & $1525,607139 \pm 53,20953$ \\
\hline Металл & $1,06 \cdot 10^{-5} \pm 2,2339 \cdot 10^{-11}$ & $11916,42018 \pm 736,08461$ \\
\hline Пенопласт & $7,29 \cdot 10^{-7} \pm 2,67196 \cdot 10^{-12}$ & $20,40046 \pm 1,57977$ \\
\hline Песок & $4,44 \cdot 10^{-7} \pm 1,35379 \cdot 10^{-13}$ & $1031,879 \pm 139,0146$ \\
\hline
\end{tabular}


вать объекты мониторинга по ТФП материалов, полученных по данным изображений с ОЭС комплексов с беспилотными летательными аппаратами.

\section{Благодарности / Acknowledgements}

Работа выполнена при финансовой поддержке РФФИ (грант № 18-08-00053А).

The reported study was funded by Russian Foundation for Basic Research (project no. 18-0800053A).

\section{Список литературы / References}

[1] Веселов Ю.Г., Данилин А.А., Тихонычев В.В. Выбор тест-объекта для оценки разрешающей способности цифровых оптико-электронных систем мониторинга земной поверхности. Наука и образование, 2012, 4. [Veselov Yu.G., Danilin A.A., Tikhonichev V.V. Select the test object for evaluation of the resolution of the digital opto-electronic systems for monitoring the earth's surface. Science and education, 2012, 4 (in Russian)]

[2] Ищук И.Н., Долгов А.А. Компьютерная модель и алгоритм построения фоноцелевой обстановки районов дистанционного мониторинга по тепловым томограммам с учетом их географического положения и метеорологических условий, Журнал Сибирского федерального университета. Техника и технологии, 2020, 13(3), 350-360 [Ishchuk I.N., Dolgov A.A. Computer model and algorithm of construction of phono-target situation of remote monitoring areas by thermal tomograms taking into account their geographical location and meteorological conditions, Journal of Siberian Federal University, Engineering \& Technologies, 2020, 13(3), 350-360 (in Russian)]

[3] Фокин К.Ф. Строительная теплотехника ограждающих частей зданий. 4-е изд., перераб. и доп. Москва: Стройиздат, 1973, с. 117. [Fokin K.F. Building heat engineering of building envelopes. 4th ed., rev. and add. Moscow: Stroyizdat, 1973, p. 117 (in Russian)]

[4] Ищук И.Н., Громов Ю.Ю., Филимонов А.М. Классификация объектов дистанционного мониторинга по тепловым томограммам, монография. Тамбов; СПб; Баку; Вена; Стокгольм; Буаке; Варна: изд-во МИНЦ «Нобелистика», 2019. 132 с. [Ischuk, I.N., Gromov Y.Y., Filimonov A.M. Classification of objects remote monitoring in thermal tomograms, a monograph. Tambov; St. Petersburg; Baku; Vienna; Stockholm; Bouake; Varna: publishing house of IINC "Nobelistika", 2019. 132 p. (in Russian)]

[5] Thermovision Tomography. [Electronic resource]. https://skygeotech.com (accessed: 31.03.2020).

[6] Šimůnek J., van Genuchten M. Th. and Šejna M. The HYDRUS-1D Software Package for Simulating the One-Dimensional Movement of Water, Heat, and Multiple Solutes in VariablySaturated Media. Version 3.0. California: Preprint Department of environmental sciences university of California riverside, 2005, $270 \mathrm{p}$.

[7] Šimůnek J., van Genuchten M. Th. and Šejna M. The HYDRUS Software Package for Simulating Two- and Three Dimensional Movement of Water, Heat, and Multiple Solutes in VariablySaturated Porous Media, Technical Manual, Version 3.0, PC Progress, Prague, Czech Republic, 2018, $274 \mathrm{p}$.

[8] Алексеев В.В., Громов Ю.Ю, Губсков Ю.А., Ищук И.Н. Методология дистанционной оценки пространственных распределений оптико-теплофизических параметров объек-

$$
-905-
$$


тов, замаскированных под поверхностью грунта. М.: ООО «Научтехлит-издат», 2014. 248 с. [Alekseev V.V., Gromov Yu.Yu., Gubskov Yu.A., Ishchuk I.N. Methodology of remote estimation of spatial distributions of optical and thermophysical parameters of objects masked under the ground surface. Moscow: OOO "Nauchtehlit-Izdat", 2014. 248 p. (in Russian)]

[9] Automation Technology. [Electronic resource]. https://www.automationtechnology.de/cms/ en/products-infrared/ (accessed: 31.03.2020). 